Note

\section{The Properties of a Tumor Necrosis Factor Mutant with the Deletion of Alanine-14}

\author{
Satoshi Nakamura, ${ }^{\dagger}{ }^{\dagger \dagger}$ Hisao Moriya, ${ }^{*}$ \\ Junji KaneKo, ${ }^{*}$ Tsukio Masegi, \\ Yoshiyuki Yamanaka, ${ }^{*}$ Keiji KozaI, ${ }^{*}$ \\ Yataro ICHIKAWA and Masako OsUMI**
}

\author{
Biotechnology Research Laboratories, \\ and * Center for Structure Analysis, \\ Teijin Limited, Hino-shi, \\ Tokyo 191, Japan \\ ** Department of Biology, \\ Japan Women's University, \\ Bunkyo-ku, Tokyo 112, Japan \\ Received June 13,'1990
}

Tumor necrosis factor (TNF) is a pleiotropic cytokine produced mainly by activated macrophages. TNF is secreted as an unglycosylated 157-amino-acid polypeptide and is thought to be trimeric in its active form. ${ }^{1)}$ Lymphotoxin (LT), a product of activated $\mathrm{T}$ lymphocytes, is a cytokine related to TNF. Both TNF and LT have similar biological properties in many respects, ${ }^{2)}$ and are recognized by the same cellular receptors. ${ }^{31}$ The amino acid sequences of the two cytokines show $28 \%$ homology, suggesting the structural and functional importance of the conserved residues. In this paper, we describe the properties of TNF mutant with the deletion of $\mathrm{Ala}^{14}$, one of the residues conserved between TNF and LT.

pTNF471 $1^{5}$ is a plasmid encoding mutant 471 . Mutant 471 (a TNF molecule with the deletion of 7 amino acids at the amino-terminal and the substitution of Pro $^{8} \mathrm{Ser}^{9}-$ Asp ${ }^{10}$ by ArgLysArg) has a higher anitumor activity and lower lethal toxicity against normal mice in comparison with intact TNF. Figure 1 shows the construction of the expression plasmid pTNF 483 encoding mutant 483 , which is mutant 471 with the deletion of $\mathrm{Ala}^{14}$.

The plasmid pTNF483 was introduced into Escherichia coli $\mathrm{C} 600 \mathrm{r}^{-} \mathrm{m}^{-}$(ATCC 33525) by the procedure of Norgard et al. ${ }^{6)}$ Culture of this transformant was done as described previously, ${ }^{7)}$ Cells were sonicated and centrifuged to obtain soluble and insoluble fractions, and the distribution of the product was analyzed by sodium dodecyl sulfate-polyacrylamide gel electrophoresis (SDSPAGE). ${ }^{8)}$ In vitro antitumor activity was measured by the dye uptake mèthod, using mouse L929 cells (ATCC CCL-929) as the target cells. ${ }^{\text {9) }}$

Mutant 471 protein (molecular weight 16,700) was produced by $E$. coli $\mathrm{C} 600 \mathrm{r}^{-} \mathrm{m}^{-}$harboring pTNF471 (E. coli [pTNF471]) as a soluble and active form in the cytoplasm, with a yield of more than $20 \%$ of the total cellular protein. ${ }^{57}$ We could detect neither mutant 483 protein nor antitumor activity in the soluble fraction of E. coli [pTNF483]. On the other hand, SDS-PAGE of the insoluble fraction of $E$. coli [pTNF483] showed a thick band at $17 \mathrm{kDa}$ (Fig. 2). Immunoblotting analysis using an anti-TNF monoclonal antibody ${ }^{10)}$ confirmed that this band corresponded to the TNF-related protein, mutant 483 (data not shown). The yield of mutant 483 was estimated to be $20-25 \%$ of the total cellular protein by densitometric analysis of SDS-PAGE.

To examine the localization of mutant 483 , electron microscopy was done. One or two high-contrast portions per cell were observed by transmission electron microscopy (TEM) and the finding suggested that mutant 483 formed insoluble inclusion bodies in the cytoplasm (Fig. 3A). High resolution scanning electron microscopy (SEM) showed a band-like swelling encircling the bacterial cell (Fig. 3B). From the SEM view of a section of $E$. coli [pTNF483], it was suggested that the band-like swelling corresponded to the position where the inclusion bodies were formed (data not shown). An E. coli [pTNF483] cell suspension was centrifuged at low speed $(500-1,000 \times g)$ after sonication to sediment inclusion bodies selectively (Fig. 3C). Inclusion bodies thus obtained contained more than $95 \%$ mutant 483 protein (Fig. 2, lane 4).

We tried to solubilize the inclusion bodies by adding $7 \mathrm{M}$ urea and to refold the mutant 483 protein by dialysis under several sets of conditions (factors examined: $\mathrm{pH}$, salt concentration, and thiol concentration). However, after mutant 483 dissolved in $7 \mathrm{M}$ urea solution, it easily re-aggregated during the refolding process. Antitumor activity could not be determined in the supernatant of a suspension of mutant 483 aggregates.

Mutant 483 , in which $\mathrm{Ala}^{14}$ was deleted from mutant 471 , was produced as insoluble inclusion bodies within $E$. coli cells and could not be converted to a soluble and active form. On the other hand, mutant 471 was produced in a soluble form at a yield as high as that of mutant 483 . Therefore, we conclude that the formation of inclusion bodies by mutant 483 was due to an intrinsic property of this protein. These suggested that the $\mathrm{Ala}^{14}$ and its neighboring residues, which are conserved in the primary structure of TNF and LT, were involved in the maintenance

$\dagger$ Present address: Department of Bioengineering, Tokyo Institute of Technology, O-okayama, Meguro-ku, Tokyo 152, Japan.

† Corresponding author. 


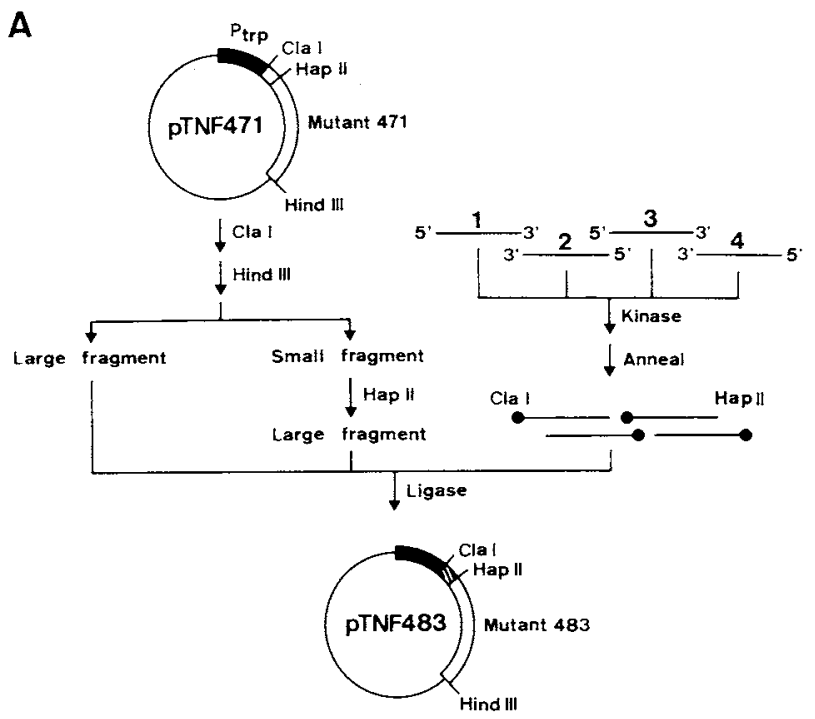

B

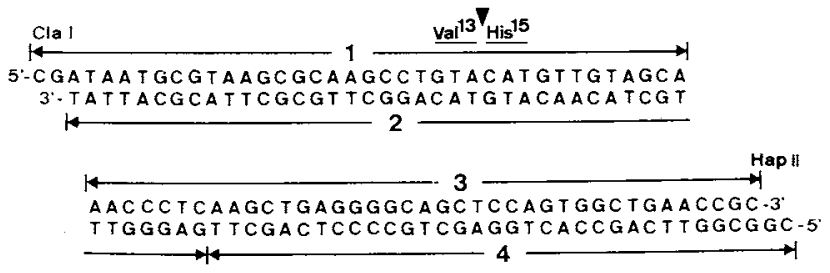

Fig. 1. Construction of pTNF483 (A) and DNA Sequences of Synthetic Oligonucleotides Used (B).

The plasmid pTNF471 was digested with ClaI and HindIII. The small ClaI-HindIII fragment of pTNF471 was then digested with HapII to obtain a HapII-HindIII fragment containing the TNF gene. Synthetic oligonucleotides containing the proposed mutation were phosphorylated, annealed, and ligated with the large ClaI-HindIII fragment of pTNF47I and the HapII-HindIII fragment of the TNF gene to yield pTNF483.

of the correct conformation of TNF. Our finding is in agreement with the report of Yamamoto et al. ${ }^{12)}$ that $\mathrm{His}^{15}$ assumes an important role in the antitumor activity of TNF. Eck and Sprang ${ }^{13)}$ have proposed, on the basis of the three-dimensional structure of TNF obtained by X-ray crystallography, that Lys ${ }^{11}$ forms an ion pair with Leu ${ }^{157}$ of the adjacent subunit in the TNF trimer. Thus, the deletion of $\mathrm{Ala}^{14}$ or the substitution of $\mathrm{His}^{15}$ may destabilize the tertiary or quaternary structure of active TNF molecules.

\section{References}

1) R. A. Smith and C. Baglioni, J. Biol. Chem., 262,
6951 (1987).

2) M. R. Ruff and G. E. Gifford, "Lymphokines," Vol. II, Academic Press, New York, 1981, pp. 235-275.

3) B. B. Aggarwal, T. E. Eessalu and P. E. Hass, Nature (London), 318, 665 (1985).

4) D. Pennica, G. E. Nedwin, J. S. Hayflick, P. H. Seeburg, R. Derynck, M. A. Palladino, W. J. Kohr, B. B. Aggarwal and D. V. Goeddel, Nature (London), 312, 724 (1984).

5) T. Masegi, S. Nakamura, M. Fukuoka, K. Kitai, A. Kato, Y. Ichikawa, M. Maeda, N. Yamauchi, N. Watanabe and Y. Niitsu, Proc. Jpn. Cancer Assoc., 1989, 279.

6) M. V. Norgard, K. Keen and J. J. Monahan, Gene,

Fig. 3. TEM View of an Ultrathin Section of $E$. coli [pTNF483] (A), SEM View of $E$. coli [pTNF483] (B), and SEM View of Inclusion Bodies (C).

Cells were fixed with $2 \%$ glutaraldehyde and $2 \%$ osmium tetroxide, dehydrated by ethanol-series, sectioned after embedding in Quetol 812, and observed with a Hitachi H-800 transmission electron microscope at $75 \mathrm{kV}$. SEM views were taken using a Hitachi S-900 high resolution scanning electron microscope at $1.5 \mathrm{kV}$, as described previously. ${ }^{11)}$ Arrows indicate the positions of inclusion bodies. Bars are $1 \mu \mathrm{m}$. 
$\begin{array}{lll}1 & 2 & 3\end{array}$

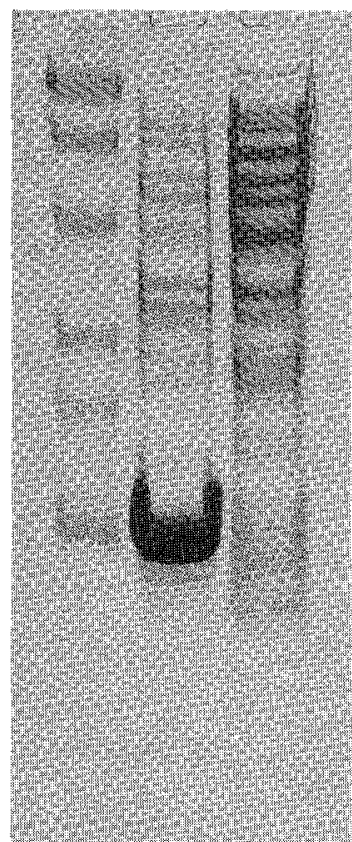

4

5

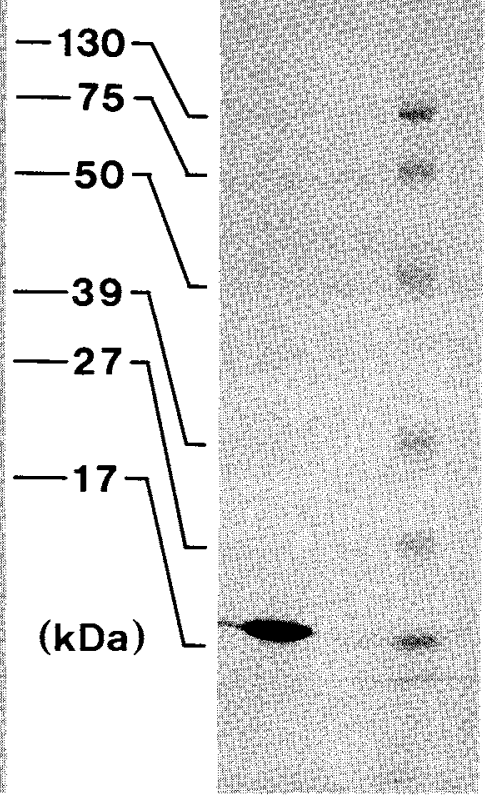

Fig. 2. SDS-PAGE Analysis of E. coli [pTNF483] and Inclusion Bodies.

SDS-PAGE was done on 15\% gel (lanes 1-3) and 10-20\% gradient gel (lanes 4,5 ) in the presence of 2-mercaptoethanol, and stained with Coomassie Brilliant Blue R-250. Lanes 1, 5, prestained molecular weight marker (Bio-Rad); lane 2, insoluble fraction of E. coli [pTNF483]; lane 3, soluble fraction of E. coli [pTNF483]; lane 4 , inclusion bodies. Each lane corresponds to $100 \mu 1$ of the original culture.
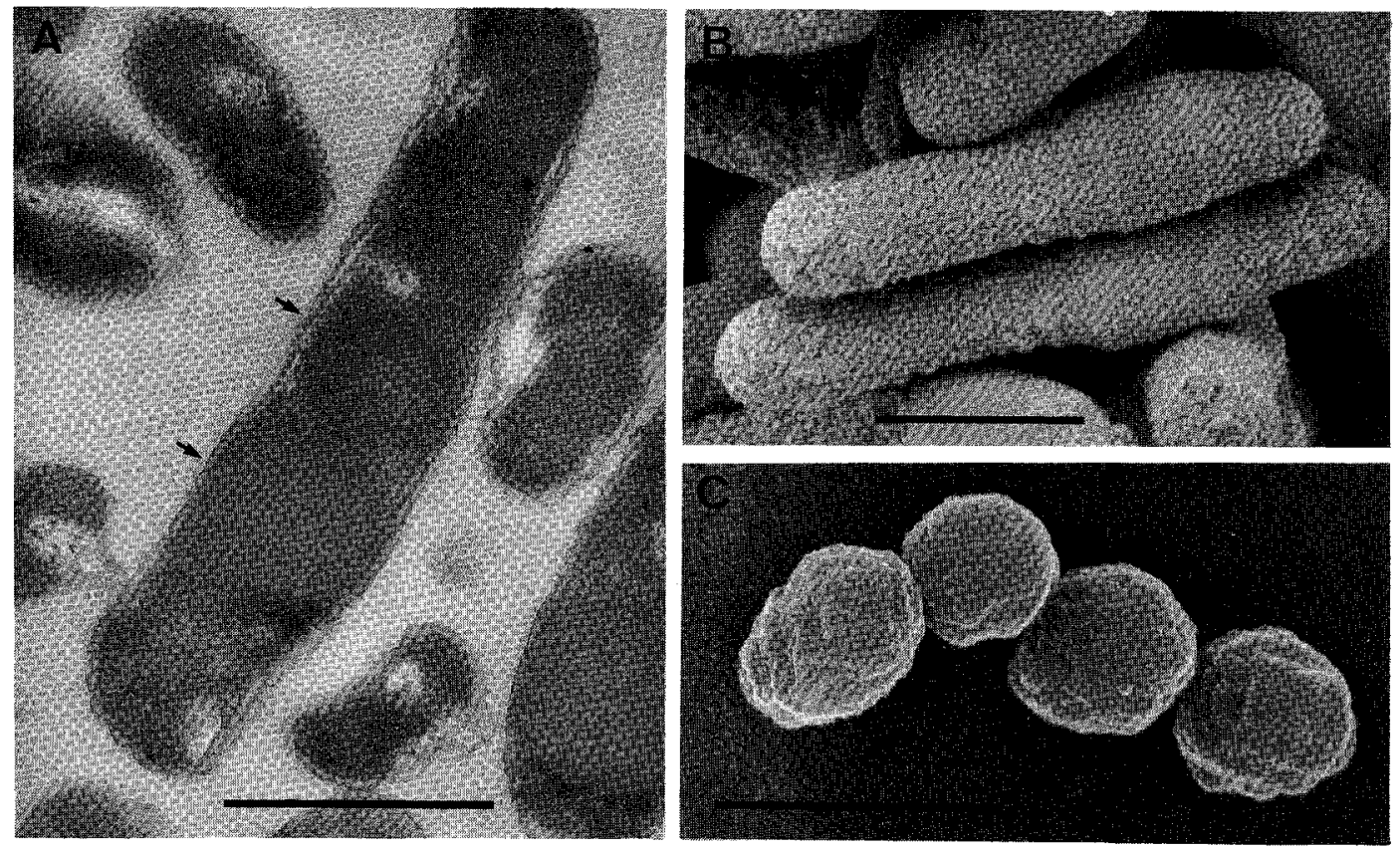
3, 279 (1978).

7) T. Masegi, S. Nakamura, K. Kitai and Y. Ichikawa, Agric. Biol. Chem., 52, 1609 (1988).

8) U. K. Laemmli, Nature (London), 227, 680 (1970).

9) S. Nakamura, T. Masegi, M. Fukuoka, K. Kitai, A. Kato, Y. Ichikawa, N. Watanabe and Y. Niitsu, Biotherapy, 4, 520 (1989).

10) K. Yone, J. Suzuki, N. Tsunekawa and S. Furukawa,
Irnmunobiology, 175, 106 (1987).

11) N. Yamada, A. Taki, M. Osumi and T. Nagatani, $J$. Electron Microsc., 37, 232 (1988).

12) R. Yamamoto, A. Wang, C. R. Vitt and L. S. Lin, Prot. Eng., 2, 553 (1989).

13) M. J. Eck and S. R. Sprang, J. Biol. Chem., 264, 17595 (1989). 